\title{
Osteochondral repair in hemophilic ankle arthropathy: from current options to future perspectives
}

\author{
ROBERTO BUDA, MARCO CAVALLO, FRANCESCO CASTAGNINI, ENRICO FERRANTI, \\ SIMONE NATALI, SANDRO GIANNINI
}

I Clinic of Orthopaedics and Traumatology, Rizzoli Orthopaedic Institute, Bologna, Italy

\begin{abstract}
Young hemophilic patients are frequently affected by ankle arthropathy. At the end stage of the disease, the current treatments are arthrodesis and arthroplasty, which have significant drawbacks. Validated procedures capable of slowing down or even arresting the progression towards the end stage are currently lacking. This review aims to discuss the rationale for and feasibility of applying, in mild hemophilic ankle arthropathy, the main techniques currently used to treat osteochondral defects, focusing in particular on ankle distraction, chondrocyte implantation, mesenchymal stem cell transplantation, allograft transplantation and the use of growth factors. To date, ankle distraction is the only procedure that has been successfully used in hemophilic ankle arthropathy. The use of mesenchymal stem cells have recently been evaluated as feasible for osteochondral repair in hemophilic patients. There may be a rationale for the use of growth factors if they are combined with the previous techniques, which could be useful to arrest the progression of the degeneration or delay end-stage procedures.
\end{abstract}

Keywords: ankle, regenerative technique, hemophilia, cartilage, chondrocyte, bone marrow concentrate.

\section{Corresponding Author:}

Francesco Castagnini, MD

I Clinic of Orthopaedics and Traumatology,

Rizzoli Orthopaedic Institute

Via Pupilli 1, 40126 Bologna, Italy

E-mail: francescocastagnini@hotmail.it

\section{Introduction}

Hemophilia is an X-linked recessive coagulopathy in which blood clotting is impaired due to deficiency of factor VIII (A type, 85\%) or factor IX (B type, 15\%) (1). Hemophilic arthropathy of the ankle remains a serious and disabling condition, with a peak of incidence in young people (1-3). Recurrent hemarthrosis causes formation of hemosiderin and iron deposits, which induces unrestrained and self-perpetuating inflammation related to reactive oxygen intermediates, abnormal neoangiogenesis and catabolic cytokines (3). Enzymatic degradation, synovial invasion and inhibition of chondrocyte matrix synthesis are the main mechanisms responsible for the development of hemophilic arthropathy $(3,4)$. The end stage of the disease is characterized mainly by the presence of abundant arthrofibrosis, muscle atrophy, inflammatory arthritis, malalignment, low bone mineral density and subchondral cysts $(3,4)$. An effective approach for preventing the onset and evolution of the arthropathy, before or soon after the first bleeding episodes, is primary prophylaxis, or, alternatively, the more cost-effective on-demand therapy $(2,5)$. Secondary prophylaxis, on the other hand, is a rapid response to an acute event, able to reduce the occurrence of bleeding episodes: nevertheless, joint degeneration is a frequently described consequence of this approach $(2,5)$.

Effective prophylaxis, entailing high costs, the development of inhibitors, and good adherence to the therapy, is hard to achieve; hence, there is still room for new prevention tools and surgical treatments $(2,5)$. Due to its good long-term results, synovectomy, using 
chemical or radioactive substances, is recommended as the first step to take in the attempt to reduce bleeding episodes (6-8). In the event of persistent hemarthrosis, a surgical synovectomy, preferably performed arthroscopically, is advisable, associated with articular debridement (6-8). Arthroscopic synovectomy with articular debridement is an effective and well tolerated treatment in children and adolescents: it is particularly recommended in cases of mild arthropathy $(7,8)$. Even though early treatment may arrest the degeneration in selected cases, sometimes the progression to degeneration cannot be arrested, either with these strategies, or by performing an osteotomy to correct malalignment. Thus, arthrodesis or arthroplasty are the necessary end-stage treatments $(6,9)$. Ankle arthrodesis, possibly performed arthroscopically, is the gold-standard technique in hemophilic ankle arthropathy, giving successful long-term results; however, the excessive mechanical stress associated with this treatment may lead to early degeneration of the midfoot and hindfoot joints $(9,10)$. In recent years, ankle replacement has been considered a valuable option, confirmed by good clinical results (in particular as regards pain control) at short-term followup $(11,12)$. That said, limited survivorship of the implant may result in the need for total ankle replacement revisions in young patients $(11,12)$.

Among the various symptomatic procedures and endstage treatments, a disease-modifying procedure is still lacking, even though such a procedure may be desirable, considering the many drawbacks of the current end-stage techniques and the young age of the patients involved. Due to the good results they have given in osteochondral lesion repair, some techniques may be suitable for treating osteochondral defects in moderate hemophilic arthropathy, with the intent of delaying or possibly avoiding recourse to end-stage procedures (13). This narrative review focuses on several new techniques used in the treatment of osteochondral defects - arthrodiatasis, autologous chondrocyte implantation (ACI), mesenchymal stem cell (MSC) transplantation, allograft transplantation and the use of growth factors (GFs) -, evaluating their current applicability and future perspectives as treatments for moderate hemophilic ankle arthropathy.

\section{Literature review}

The current English language literature on ankle distraction, ACI, MSC transplantation, allograft transplantation and GFs, present in the PubMed database, was reviewed to assess the potential for using these techniques in hemophilic joints. The database was systematically searched for information on the outcomes of these techniques in ankle degenerative disease and osteoarthritis, in order to evaluate their feasibility and applicability in degenerated inflamed ankle joints. The rationale for this strategy was the evidence, detailed in a review by Valentino (3), of similar pathogenetic features in hemophilic arthropathy and osteoarthritis. The reference lists of the papers were also analyzed for further data sources. At present there appear to be only two published papers that strictly deal with hemophilia and the aforementioned techniques: the work by Von Meegeren et al. on arthrodiatasis and the study on MSC transplantation by Ebihara et al. $(14,15)$.

\section{Ankle distraction}

Ankle distraction has recently been used to treat three young patients with hemophilic arthropathy of the ankle, achieving clinical and radiological improvement at short-term follow-up, with no significant blood loss (14). The Authors considered this application justified by the noteworthy clinical outcomes of previous studies in ankle osteoarthritis. In a work by Ploegmaker et al., joint distraction achieved good and durable clinical results $(73 \%$ of the cases) in post-traumatic ankle osteoarthritis at a minimum follow-up of seven years (16). In a randomized controlled trial, ankle distraction outcomes at short-term follow-up were superior to the outcomes obtained in a debridement control group, with distraction found to result in lower subchondral bone sclerosis and higher cartilage thickness (17). A paper on CT examination at two-year follow-up after joint distraction and articular debridement highlighted a normalization of subchondral bone density, with reduction of cysts and sclerosis; cartilage thickness was also found to be improved (18). In another study by the same Authors, in which they treated osteoarthritic knees with distraction, an analysis of collagen II biomarkers was positive for collagen synthesis, suggesting the presence of hyaline-like cartilage restoration at short-term follow up (19). Beyond the improvements 
linked to mechanical interventions, an in vitro study highlighted a higher presence of anabolic GFs (TGFbeta, BMPs) than of catabolic cytokines (TNFa e IL1); the Authors also found that intermittent fluid movement was able to promote matrix synthesis by chondrocytes and MSC (20). Nevertheless, ankle distraction should not be expected to provide a quick recovery, as the device needs to be applied for at least two-three months and clinical benefits are not reported before six months (21). Moreover, Saltzmann et al. reported a moderate rate of infectious and neurological complications with this treatment (21).

\section{Autologous chondrocyte implantation}

It has been suggested that ACI might constitute a possible treatment of osteochondral defects in hemophilia, but to date in vitro and in vivo applications are still lacking (13). ACI was introduced for the repair of pure osteochondral lesions of the knee in 1987 by Brittberg, after which many technical tips were advanced to refine the procedure (22). Comparison of outcomes between osteochondral lesions treated with ACI and lesions treated using other techniques, namely mosaicplasty and microfractures, showed no substantial difference (22). The results of ACI in osteochondral lesions of the ankle have been described as encouraging on the basis of biopsy studies showing hyaline cartilage restoration, and MRI quality evaluation (23). Osteoarthritis is traditionally considered one of the main limits of the ACI technique $(22,24,25)$. Filardo et al., studying matrixassisted chondrocyte transplantation in early osteoarthritic knees, found that the procedure resulted in poor patient satisfaction, low clinical and functional outcomes and a high rate of failures $(27 \%)$ at mid-term follow-up (25). Conversely, other papers support the use of ACI in osteoarthritis: Minas et al., in a study of 155 knees of young people with early osteoarthritis, showed that $92 \%$ of patients had no need of arthroplasty at five years (26). This latter finding was substantially in line with work by Desando et al. in an animal model: rabbit early-osteoarthritic knees, treated with a hyaluronic scaffold and ACI, showed histological improvement of the osteochondral layer, with a significant presence of collagen II. However, early intervention was considered mandatory (27).

\section{Autologous mesenchymal stem cell transplantation}

Although there has, as yet, been no clinical application of MSC transplantation in hemophilia, in recent years the possibility of concrete application of the technique in this setting has been generating considerable interest $(13,15)$ (Fig. 1). The logic for performing autologous MSC transplantation is that the technique exploits the regenerative potential of pluripotent cells and allows the restoration of an osteochondral layer that, displaying hyaline-like features, is very similar to the previous, worn one. MSC transplantation has been used in many trials and studies focusing on osteochondral lesions, giving remarkable results characterized by the regeneration of hyaline-like tissue, even in ankle defects $(28,29)$. Like ACI, MSC transplantation has been considered unsuitable as a treatment for osteoarthritis due to the deleterious effects of inflammation on MSC (30). Nevertheless, a rationale for the application of MSC in osteoarthritic joints has recently been shown (31). Interesting results in osteoarthritis have been provided by an animal model, where MSC were applied on a scaffold, and by in vivo studies, where MSC were injected $(32,33)$. To date, the best quality research into the possible use of MSC in osteoarthritic joints is that performed by Wakitani et al. (34). They applied autologous expanded mesenchy-

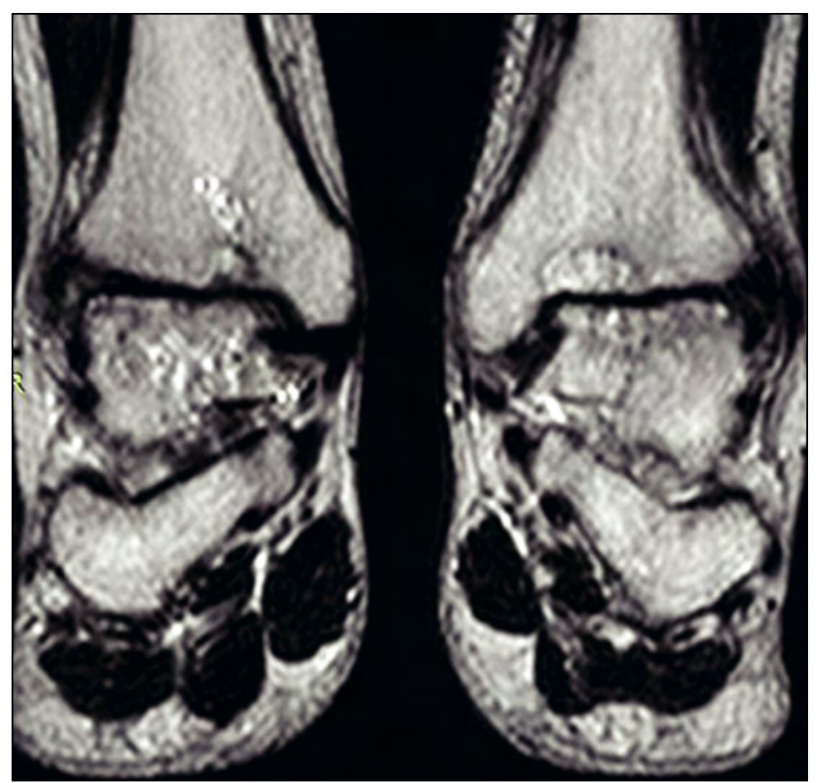

Fig. 1. A coronal MRI scan of bilateral hemophilic ankle arthropathy in a 24-year-old man, affected by hemophilia type B. It is possible to observe whole joint degeneration with extensive involvement of the osteochondral layer and joint space reduction. This patient underwent autologous mesenchymal stem cell transplantation as a salvage procedure. 
mal cells (a two-step approach) with a collagen gel during high tibial osteotomy in varus osteoarthritic knees; the results were compared with those obtained in a cell-free group treated with microfractures, or abrasion, and realignment. After 42 weeks, the second-look arthroscopic evaluation highlighted the presence of soft cartilage with some hyaline-like features in the MSC group, but not in the control group. In line with Wakitani et al., Veronesi et al. concluded that the two-step approach may be more desirable in degenerated joints, as the extracellular matrix may protect the cells from the inflammatory environment $(28,34)$. Given the good results obtained in arthritic knees, and with a view to starting a clinical trial, Ebihara et al. studied the feasibility of autologous MSC in hemophilic patients (15). Three patients underwent a bone marrow aspiration from the iliac crest. When cultured with autologous serum and expanded, MSC were found to display phenotypic and genotypic features similar to those of the cells of healthy individuals, including the capacity to differentiate into a chondrogenic lineage. The number of cells per $\mathrm{mL}$ sufficient to achieve adequate repair of osteochondral defects in osteoarthritic joints was established according to previously stated parameters (35).

\section{Allograft transplantation}

In advanced centers, allograft transplantation has recently become a remarkably popular technique, due to the lack of quality studies on the previously described techniques; furthermore, allograft transplantation has the noteworthy advantage of being able to fill massive osteochondral defects without the need for autologous cells or tissue (36). Nevertheless, no in vitro or in vivo works are currently available on allograft transplantation in hemophilia. In a study by Haene et al. (36), 17 ankles underwent fresh osteochondral allograft transplantation for large and deep defects and were clinically and radiologically evaluated at four-year follow-up. Four of the 17 ankles had excellent outcomes; five failed. Radiological evaluation showed several cases of degenerative signs, even in other parts of the ankle joint. In a study by Bugbee et al. (37), in which 88 ankles underwent bipolar fresh osteochondral transplantation to treat symptomatic osteoarthritis, the survivorship of the allograft was estimated to be $76 \%$ at 5 years and $44 \%$ at 10 years. This longlasting survivorship may have been facilitated by colo- nization of the allograft by host cells, improving its integration (38). Nevertheless, in Bugbee's report, the clinical failure rate stood at $29 \%$, which is higher than that of arthrodesis or arthroplasty case series (37).

\section{Growth factors}

GFs have been intensively used in recent years, due to their anabolic effects on chondrocytes, synoviocytes, bone marrow-derived cells and matrix production in general; however, there has been no work focusing specifically on hemophilia (39). Mei-Dan et al., in a randomized control trial, studied the administration of platelet-rich plasma (PRP) and hyaluronic acid in osteochondral lesions of the talus, and reported good clinical results at six months (40). In a review by Filardo et al., the treatment of knee chondral damage with PRP resulted in limited and not durable improvements, moreover mostly restricted to young people with only modest signs of osteoarthritis (41). Recently, GFs have been used along with reparative-regenerative techniques for osteochondral state improvement. In a work by Lee et al., two groups of patients older than 40 years were treated with microfractures for osteochondral lesions of the knee (42). The group in which PRP was administered intra-operatively had better clinical results, with a better quality of cartilage at the second-look arthroscopy (harder and thicker than in the control group). Similarly, platelet rich fibrin has been administered after MSC transplantation in osteochondral lesions of the talus, in order to promote cell differentiation and implant stability (29). A more modern and futuristic perspective was provided by Gelke et al., who showed that gene therapy, along with chondrogenic progenitor implantation, may be a way of enhancing anti-inflammatory cytokine expression, in order to improve osteochondral regeneration (43). This approach may improve the inflammatory, degenerative joint environment after a traumatic insult. The introduction of an antiinflammatory and GF therapy along with matrix-assisted chondrocyte implantation was proposed as a possible procedure in joint degeneration, able to protect cells and stimulate the restorative process (44).

\section{Discussion}

End-stage procedures in hemophilic arthropathy may be poorly accepted by young patients and can present significant drawbacks, for example progression of 
midfoot or subtalar joint degeneration or limited survivorship of the prosthesis (9-12). There is currently increasing interest in the restoration of osteochondral defects in moderate ankle hemophilic arthropathy using novel procedures (13). The current literature describes several promising techniques, but only for focal osteochondral lesions, given that joint degeneration, large defect size and hemophilic arthropathy are all considered contraindications (13). Nevertheless, given the scarcity of available literature and the fact that no minimally invasive procedure has been validated for hemophilic arthropathy, it seems opportune to critically analyze the state-of-the art techniques for osteochondral repair also from the perspective of their possible application in this disease. The aim of treatment is to reduce the highly catabolic environment of affected joints, which is responsible for cartilage degeneration, in order to slow down, and hopefully arrest, the progression of arthritis. Among the more recent procedures with a regenerative aim, chondrocyte implantation is one of the most widely adopted and it has been shown to be successful for osteochondral lesion repair (22). Conversely, according to the current literature, degenerated joints do not seem to benefit from this approach (25). Since hemophilic arthropathy induces a complex, degenerated and inflamed joint environment, ACI may not be effective if applied to treat joint degeneration in hemophilia (25). If used with the aim of controlling the progression of osteoarthritis, ACI could nevertheless have a role in the very early stage of the disease, i.e. in the treatment of the focal, isolated osteochondral lesions in hemophilic ankles with no signs of arthropathy.

Allograft transplantation is a technique used to replace the ankle osteochondral layer. When assessing whether allograft transplantation is indicated, it should be born in mind that it has a considerable failure rate, particularly in large defects and bipolar graft transplantation $(36,37)$; moreover, it is a technically demanding, open-field technique associated with significant tissue trauma, which remains a relative contraindication in hemophilia. It may be indicated in hemophilic ankle arthropathy only as a revision surgery procedure after failed restoration of the osteochondral layer, in patients unwilling to undergo arthrodesis and arthroplasty.

The current literature seems to support the use of ankle distraction and MSC transplantation in degene- rated joints in pursuit of a whole-joint approach given that they can produce inflammatory status improvement and osteocartilaginous layer repair. With regard to hemophilia, ankle distraction showed encouraging short-term clinical and radiological results in three hemophilic patients affected by ankle arthropathy (14). Even though the evidence of hyaline cartilage regeneration still needs to be clarified and strengthened, this technique can currently be considered part of the treatment paradigm for hemophilic arthropathy $(14,19,21)$.

The good outcome in osteochondral lesions and more recently in osteoarthritis has prompted consideration of the use of MSC in hemophilia, as it offers the possibility of restoring a good clinical status and repairing osteochondral defects while exploiting the associated anti-inflammatory effect of MSC in the joint environment $(31,34)$. Ebihara et al. showed the feasibility using of MSC in hemophilic patients, highlighting the chondrogenic potential of these cells (15).

Nevertheless, clinical application in hemophilic joints is still lacking, as is histological evidence of hyaline regeneration in degenerated joints (Fig. 2).

The use of GFs is a very interesting approach, both in conservative and surgical treatments (41). A fascinating approach may be the integration of GFs with previous techniques, as a means of enhancing cell differentiation and proliferation. Another stimulating future perspective for hemophilic patients may be offered by gene therapy, used as a means of conveying some

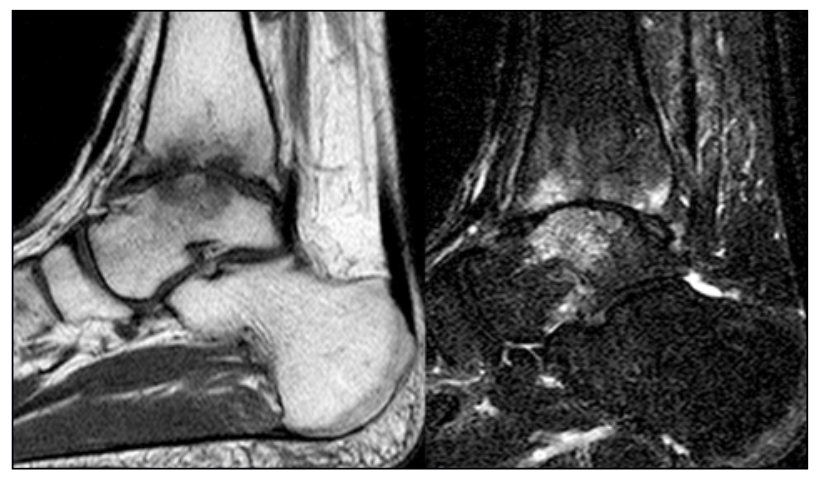

Fig. 2. Two sagittal MRI scans of hemophilic ankle arthropathy in a 27-year-old man treated with a large debridement, synovectomy and autologous mesenchymal stem cell transplantation using the "onestep" technique, after six months. Few signs of osteochondral layer regeneration can be identified, but a large subchondral edema was still evident: the patient had quite an encouraging clinical outcome (AOFAS score: 77 points). 
anti-inflammatory cytokines to inflamed joints (44). Young hemophilic patients with mild signs of ankle degeneration in whom other more conservative approaches fail are ultimately addressed to end-stage treatments, even though these have many drawbacks. There is now evidence coming from the literature on osteoarthritis which suggests that promising new techniques with regenerative purposes may find application in hemophilic arthropathy; these include, in particular, ankle distraction and autologous MSC transplantation, on account of their capacity to control degenerative and inflammatory processes. Both techniques may benefit from the application of GFs, and in the future from the development of gene therapy. Even though hemophilic arthropathy is a rare condition, there is a real need for scientific studies able to answer the various questions arising from the literature and ultimately provide an effective solution for hemophilic patients with arthropathy.

\section{References}

1. Luck JV Jr, Silva M, Rodriguez-Merchan EC, et al. Hemophilic arthropathy. J Am Acad Orthop Surg. 2004;12: 234-245.

2. Schobess R, Kurnik K, Friedrichs F, et al. Effects of primary and secondary prophylaxis on the clinical expression of joint damage in children with severe haemophilia A. Results of a multicenter non-concurrent cohort study. Thromb Haemost. 2008;299:71-76.

3. Valentino LA. Blood-induced joint disease: the pathophysiology of hemophilic arthropathy. J Thromb Haemost. 2010; 8:1895-902.

4. Dunn AL. Pathophysiology, diagnosis and prevention of arthropathy in patients with haemophilia. Haemophilia. 2011;17:571-578.

5. Manco-Johnson MJ, Abshire TC, Shapiro AD, et al. Prophylaxis versus episodic treatment to prevent joint disease in boys with severe hemophilia. N Engl J Med. 2007;357:535544.

6. Pasta G, Forsyth A, Merchan CR, et al. Orthopaedic management of haemophilia arthropathy of the ankle. Haemophilia. 2008;14 Suppl 3:170-176.

7. Dunn AL, Busch MT, Wyly JB, et al. Arthroscopic synovectomy for hemophilic joint disease in a pediatric population. J Pediatr Orthop. 2004;24:414-426.

8. Rodriguez-Merchan EC. Ankle surgery in haemophilia with special emphasis on arthroscopic debridement. Haemophilia. 2008;14:913-919.

9. Fong JY, Luck JV, Silva M. Ankle fusion in hemophilia. San Diego, CA: American Academy of Orthopaedic Surgery, Annual Meeting. 2007.

10. Tsukamoto S, Tanaka Y, Matsuda T, et al. Arthroscopic ankle arthrodesis for hemophilic arthropathy: two cases report. Foot (Edinb). 2011;21:103-105.

11. Radossi P, Bisson R, Munari F, et al. Total ankle replacement for end-stage arthropathy in patients with haemophilia. Haemophilia. 2008;14:658-660.

12. Barg A, Elsner A, Hefti D, et al. Haemophilic arthropathy of the ankle treated by total ankle replacement: a case series. Haemophilia. 2010;1;16:647-655.

13. Liras A, Gaban AS, Rodriguez-Merchan EC. Cartilage restoration in haemophilia: advanced therapies. Haemophilia. 2012;18:672-679.

14. van Meegeren ME, van Vulpen LF, Roosendaal G, et al. Joint distraction: a treatment to consider for haemophilic arthropathy. Haemophilia. 2012;18:e418-e420.

15. Ebihara Y, Takedani H, Ishige I, et al. Feasibility of autologous bone marrow mesenchymal stem cells cultured with autologous serum for treatment of haemophilic arthropathy. Haemophilia. 2013;19:e87-e89.

16. Ploegmakers JJ, van Roermund PM, van Melkebeek J, et al. Prolonged clinical benefit from joint distraction in the treatment of ankle osteoarthritis. Osteoarthritis Cartilage. 2005;13:582-588.

17. Marijnissen AC, Van Roermund PM, Van Melkebeek J, et al. Clinical benefit of joint distraction in the treatment of severe osteoarthritis of the ankle: proof of concept in an open prospective study and in a randomized controlled study. Arthritis Rheum. 2002;46:2893-2902.

18. Intema F, Thomas TP, Anderson DD, et al. Subchondral bone remodeling is related to clinical improvement after joint distraction in the treatment of ankle osteoarthritis. Osteoarthritis Cartilage. 2011;19:668-675.

19. Intema F, Van Roermund PM, Marijnissen AC, et al. Tissue structure modification in knee osteoarthritis by use of joint distraction: an open 1-year pilot study. Ann Rheum Dis. 2011;70:1441-1446.

20. van Valburg AA, van Roy HL, Lafeber FP, et al. Beneficial effects of intermittent fluid pressure of low physiological magnitude on cartilage and inflammation in osteoarthritis. An in vitro study. J Rheumatol. 1998;25:515-520.

21. Saltzman CL, Hillis SL, Stolley MP, et al. Motion versus fixed distraction of the joint in the treatment of ankle osteoarthritis: a prospective randomized controlled trial. J Bone Joint Surg Am. 2012;94:961-970.

22. Vasiliadis HS, Wasiak J. Autologous chondrocyte implantation for full thickness articular cartilage defects of the knee. Cochrane Database Syst Rev. 2010;10:CD003323.

23. Battaglia M, Vannini F, Buda R, et al. Arthroscopic autologous chondrocyte implantation in osteochondral lesions of the talus: mid-term T2-mapping MRI evaluation. Knee Surg Sports Traumatol Arthrosc. 2011;19:1376-1384.

24. Nehrer S, Dorotka R, Domayer S, et al. Treatment of fullthickness chondral defects with Hyalograft $C$ in the knee: a prospective clinical case series with 2 to 7 years' follow-up. Am J Sports Med. 2009;37:81S-87S.

25. Filardo G, Vannini F, Marcacci M, et al. Matrix-assisted autologous chondrocyte transplantation for cartilage regeneration in osteoarthritic knees: results and failures at midterm followup. Am J Sports Med. 2013;41:95-100.

26. Minas T, Gomoll AH, Solhpour S, et al. Autologous chondrocyte implantation for joint preservation in patients with early osteoarthritis. Clin Orthop Relat Res. 2010;468:147-157.

27. Desando G, Cavallo C, Tschon M, et al. Early-term effect of adult chondrocyte transplantation in an osteoarthritis animal model. Tissue Eng Part A. 2012;18:1617-1627.

28. Veronesi F, Giavaresi G, Tschon M, et al. Clinical use of bone marrow, bone marrow concentrate and expanded bone marrow mesenchymal stem cells in cartilage disease. Stem Cells Dev. 2013;22:181-192.

29. Giannini S, Buda R, Battaglia M, et al. One-step repair in talar 
osteochondral lesions: 4-year clinical results and t2-mapping capability in outcome prediction. Am J Sports Med. 2013; 41:511-518.

30. Murphy JM, Dixon K, Beck S, et al. Reduced chondrogenic and adipogenic activity of mesenchymal stem cells from patients with advanced osteoarthritis. Arthritis Rheum. 2002;46:704-713.

31. Qi Y, Feng G, Yan W. Mesenchymal stem cell-based treatment for cartilage defects in osteoarthritis. Mol Biol Rep. 2012; 39:5683-5689.

32. Grigolo B, Lisignoli G, Desando G, et al. Osteoarthritis treated with mesenchymal stem cells on hyaluronan-based scaffold in rabbit. Tissue Eng Part C Methods. 2009;15:647-658.

33. Orozco L, Munar A, Soler R, et al. Treatment of knee osteoarthritis with autologous mesenchymal stem cells: a pilot study. Transplantation. 2013;95:1535-1541.

34. Wakitani S, Imoto K, Yamamoto T, et al. Human autologous culture expanded bone marrow mesenchymal cell transplantation for repair of cartilage defects in osteoarthritic knees. Osteoarthritis Cartilage. 2002;10:199-206.

35. Wakitani S, Okabe T, Horibe S, et al. Safety of autologous bone marrow-derived mesenchymal stem cell transplantation for cartilage repair in 41 patients with 45 joints followed for up to 11 years and 5 months. J Tissue Eng Regen Med. 2011;5:146-150.

36. Haene R, Qamirani E, Story RA, et al. Intermediate outcomes of fresh talar osteochondral allografts for treatment of large osteochondral lesions of the talus. J Bone Joint Surg Am. 2012;94:1105-1110.
37. Bugbee WD, Khanna G, Cavallo M, et al. Bipolar fresh osteochondral allografting of the tibiotalar joint. J Bone Joint Surg Am. 2013;95:426-432.

38. Neri S, Vannini F, Desando G, et al. Ankle bipolar fresh osteochondral allograft survivorship and integration: transplanted tissue genetic typing and phenotypic characteristics. J Bone Joint Surg Am. 2013;95:1852-1860.

39. Fortier LA, Barker JU, Strauss EJ, et al. The role of growth factors in cartilage repair. Clin Orthop Relat Res. 2011; 469:2706-2715.

40. Mei-Dan O, Carmont MR, Laver L, et al. Platelet-rich plasma or hyaluronate in the management of osteochondral lesions of the talus. Am J Sports Med. 2012;40:534-541.

41. Filardo G, Kon E, Roffi A, et al. Platelet-rich plasma: why intra-articular? A systematic review of preclinical studies and clinical evidence on PRP for joint degeneration. Knee Surg Sports Traumatol Arthrosc. 2015;23:2459-2474.

42. Lee GW, Son JH, Kim JD, et al. Is platelet-rich plasma able to enhance the results of arthroscopic microfracture in early osteoarthritis and cartilage lesion over 40 years of age? Eur J Orthop Surg Traumatol. 2013;23:581-587.

43. Gelse K, von der Mark K, Schneider H. Cartilage regeneration by gene therapy. Curr Gene Ther. 2003;3:305-317.

44. Schulze-Tanzil G. Activation and dedifferentiation of chondrocytes: implications in cartilage injury and repair. Ann Anat. 2009;191:325-338. 